\title{
The Effect of Si Impurity at the Al $\Sigma 5$ Grain Boundary: a First Principle Computational Tensile Test Study
}

\author{
Jiuhui $L I^{1)}$, Xing $Z H A O^{1) \dagger}$, Dongsheng $W A N G^{2)}$ and Fanshun $M E N G^{1)}$ \\ 1) College of Science, Liaoning University of Technology, Jinzhou 121001, China \\ 2) College of Science, Yanshan University, Qinhuangdao 066004, China \\ [Manuscript received 5 April 2013, in revised form 5 August 2013] \\ (c) The Chinese Society for Metals and Springer-Verlag Berlin Heidelberg
}

First principle computational tensile tests (FPCTT) are performed to the Al $\Sigma 5$ grain boundaries (GBs) with and without substitution or interstitial Si impurity. The obtained stress-strain relationships and atomic configurations demonstrate that the $\mathrm{Al} \Sigma 5 \mathrm{GBs}$ with and without substitutional or interstitial Si impurity show different fracture modes. The mechanisms of the different fracture modes are analyzed based on the charge density and the density of states. The results show that the charge redistributions of the atoms in the vicinity of GBs and the covalent interactions between $\mathrm{Si}$ and its neighboring $\mathrm{Al}$ atoms determine the fracture modes.

KEY WORDS: Al $\Sigma 5$ GB; Si impurity; First principle computational tensile test

\section{Introduction}

Al-Si alloys have been commercially used to produce the engine block due to their high strength to weight ratio ${ }^{[1]}$. The engine block works under mechanical stresses due to its relative motion to other engine parts. High fatigue strength is critical to the engine life. The whole fatigue process largely depends on the microcrack initiation and propagation. The microcrack occurs relatively easily at the grain boundary of the pure metal and alloys. Therefore, it is necessary to understand the mechanical responds of the grain boundary to the external load and the changes of internal stress and strain fundamentally. The mechanical properties of polycrystalline with GBs can be studied experimentally. However, the mechanical behaviors of GBs are highly correlated with the electronic structure. It is difficult to get the detail information at the atomic and electronic level from experimental measurements. On the other hand, first principle calculations based on electronic structure theory may provide some fundamental information on the mechanical properties of $\mathrm{GBs}^{[2-9]}$.

† Corresponding author. Prof., Ph.D.; Tel.: +86 416 4198716; E-mail address: zhao-heng-xing@126.com (Xing ZHAO)

DOI: $10.1007 / \mathrm{s} 40195-013-0083-2$
The mechanical properties of $\mathrm{Al}$ GBs have been studied in recent years by using first principle methods. Lu et al. ${ }^{[10]}$ investigated pure Al $\Sigma 9$ GB by using the ab initio tensile test. They found that strong local bonds are formed around the defects and the boundary is still strong due to the interface reconstruction. Zhang et al. ${ }^{[11]}$ revealed that the fracture of Al $\Sigma 9$ GB model can be characterized by the grain boundary interfacial-reconstruction. They found that the reconstructed bonds are somehow covalent characterized, which results in rather high grain boundary strength in comparison with that of the bulk Al. Pang et al. ${ }^{[12]}$ studied the shear properties of $\mathrm{Al}$ single crystals and grain boundaries and find that there is pronounced easy-sliding direction along the tilt axis for the [110] tilt grain boundaries. The theoretical shear strength scales with the height of the slip barriers and exhibits a relation with the misorientation angle: the closer the angle to $90^{\circ}$, the higher the shear stress. Lu et al. ${ }^{[13]}$ investigated the effects of impurities on Al $\Sigma 9$ GB and conclude that different impurities may have various effects on the local atomic and electronic structures of the grain boundaries, which dominates the mechanisms of embrittlement. Liu et al. ${ }^{[14]}$ studied Sr segregation at $\mathrm{Al} \Sigma 9(2 \overline{2} 1) /[110]$ grain boundary and the results showed that the boundary expands and the charge density decreases significantly, which 
results in the weakness of the grain boundary.

In this study, $\mathrm{Al} \Sigma 5(012) /[100]$ symmetrical tilt $\mathrm{GB}$ is chosen to study the GBs in $\mathrm{Al}$ since it is the high energy GB, which tends to break before low-energy ones $^{[15]}$. The stress-strain curves and the configurations of the atoms are calculated by using a first principles method. The results demonstrate that the $\mathrm{Al}$ $\Sigma 5$ GB with and without substitution or interstitial Si impurity shows different fracture modes. In order to determine the electronic origin of the different fracture modes, we examine the charge density distribution and local density of states, based on which we analyze the interactions of $\mathrm{Si}$ with $\mathrm{Al}$ atoms to investigate deeply mechanisms of different fracture modes of the GBs models.

\section{Computational Method and Model}

The calculations are carried out by using the Vienna ab-initio Simulation Package $\left(\right.$ VASP ${ }^{[16-19]}$ based on the density functional theory (DFT).The projector augmented wave method ${ }^{[20,21]}$ is used. The generalized gradient approximation ${ }^{[22]}$ parameterized by Perdue-Burke-Ernzerh is employed to describe the electron correlation and exchange energy. The plane wave cut off is set to $275 \mathrm{eV}$. The Brillouin zone is sampled with a Monkhorst Pack $5 \times 2 \times 1$ $k$-point mesh. The first-order Methfessel-Paxton method with a width of $0.1 \mathrm{eV}$ is adopted for the Fermi surface smearing. Geometry optimization is performed by using the conjugated-gradient minimization scheme. The convergence tolerance is set to $1.0 \times 10^{-5} \mathrm{eV} /$ atom for the total energy and $1.0 \times 10^{-2} \mathrm{eV} / \mathrm{nm}$ for the inter-atomic force.

In this study, the Al $\Sigma 5(012) /[100]$ symmetric tilt GB containing 20 layers with two atoms per layer is constructed by first cleaving an fcc Al lattice along a (012) plane. Then, one half of the separated crystal is rotated $36.9{ }^{\circ} \mathrm{C}$ about an axis normal to (100) plane. Re-joining the two grains after removing the overlapping boundary atoms produces the desired $\Sigma 5$ structure as shown in Fig. 1, in which one in five lattice points are coincident. The lattice constant of fcc $\mathrm{Al}$ reproduced by our GGA calculation is $0.407 \mathrm{~nm}$. The sizes of the GB model are $0.407 \mathrm{~nm}$ in the [100] direction, $0.904 \mathrm{~nm}$ in the [021] direction and $1.808 \mathrm{~nm}$ in the [012] direction. The 20-layer supercell model (in [012] direction) is large enough to neglect the interaction between the two neighboring GBs caused by the periodic supercell models according to the energy calculations of the supercell models with different dimensions in [012] direction.

\section{Results and Discussion}

\subsection{Stress-strain and atomic configurations}

In the initial optimization of the system the atomic positions have been relaxed with the fixed shape and

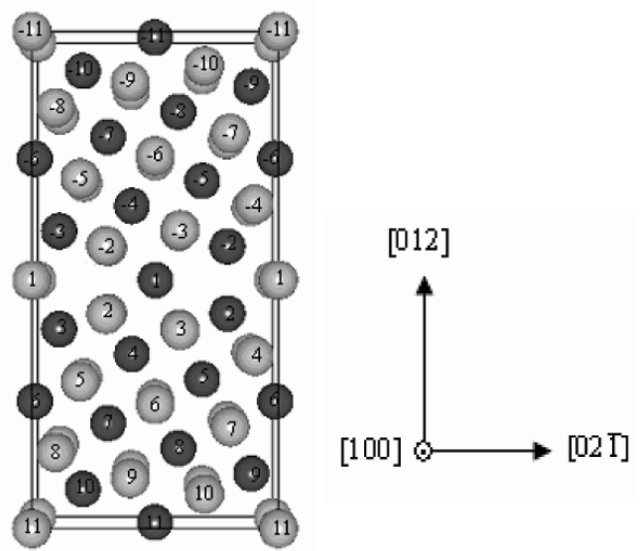

Fig. 1 Supercell model of Al $\sum 5(012) /[100]$ GB containing $40 \mathrm{Al}$ atoms (The grey and dark grey balls represent $\mathrm{Al}$ atoms in the (100) and (200) planes along the [100] direction, respectively. The atomic sites are labeled by numbers counted from the GB plane. Site 1 is on the mirror plane, site $i$ and site $-i(i=2-11)$ have mirror symmetry)

dimensions of the supercell. In the sequent tensile process we change the dimension of the supercell in [012] direction with the fixed dimensions in the two other directions and make full relaxation of the atomic positions.

Fig. 2 shows the stress-strain curves for the $\mathrm{Al}$ GB without Si impurity, Al GB with substitutional $\mathrm{Si}$, and $\mathrm{Al} \mathrm{GB}$ with interstitial Si. The tensile stress is in the [012] direction.

In the pure Al GB model, the tensile stress appears beyond the strain of $6 \%$ because of the residual compressive stress exists in the original supercell. With the increase of the strain, the tensile stress increases almost linearly until the strain reaches $22 \%$. The following stress keeps constant when the strain changes from $24 \%$ to $34 \%$. The stress drops suddenly at the strain rang of $36 \%$ to $38 \%$ indicating that the model of the pure $\mathrm{Al} \mathrm{GB}$ fracture occurs.

Site 1 (labeled by -1 in Fig. 1) and site 2 (labeled by -2 ) are the optional sites for the substitutional impurity in the Al GB. The relative stability of Si site in the GB is determined with the formation energy of $\mathrm{Si}$, which is calculated by

$$
E_{\mathrm{GB}-\mathrm{Si}}^{\mathrm{f}}=E_{\mathrm{GB}-\mathrm{AlSi}}^{\mathrm{T}}-E_{\mathrm{GB}-\mathrm{Al}}^{\mathrm{T}}+E_{\mathrm{Al}}-E_{\mathrm{Si}}
$$

$E_{\mathrm{GB}-\mathrm{AlSi}}^{\mathrm{T}}, E_{\mathrm{GB}-\mathrm{Al}}^{\mathrm{T}}, E_{\mathrm{Al}}$ and $E_{\mathrm{Si}}$ are the energies of the Al-Si GB supercell, pure Al GB supercell, per Al atom in fcc bulk $\mathrm{Al}$ and an isolated $\mathrm{Si}$ atom, respectively. The deformation energies are $-4.673 \mathrm{eV}$ and $-4.185 \mathrm{eV}$ for the substitutional Si at site 2 and site 1 , respectively. Therefore, the relatively stable site of $\mathrm{Si}$ atom is site 2 in GB.

In the $\mathrm{Al} \mathrm{GB}$ model with the substitutional impurity, Al atom in site 2 of (100) plane is replaced 


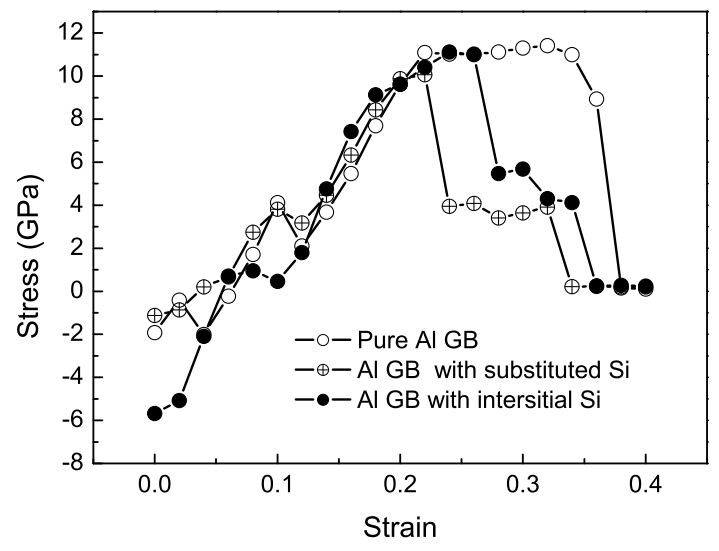

Fig. 2 Stress-strain curves for the pure Al GB model, Al GB model with substitution Si and Al GB model with interstitial $\mathrm{Si}$

by $\mathrm{Si}$ atom (nominating $\mathrm{Si}(2)$ and the rest may be inferred). The tensile stress increases almost linearly until the strain reaches $22 \%$. The stress decreases greatly when the strain changes from $22 \%$ to $24 \%$, which implies that the local fracture occurs. When the strain changes from $32 \%$ to $34 \%$ the stress drops suddenly indicating that the complete fracture occurs.

For the Al GB with interstitial Si at the (200) mirror plane, where the $\mathrm{Si}$ atom is surrounded by $\mathrm{Al}(2)$ and $\mathrm{Al}(-2)$ in the (100) plane and $\mathrm{Al}(3)$ and $\mathrm{Al}(-3)$ in the (200) plane, the stress decreases greatly when the strain changes from $26 \%$ to $28 \%$, which implies that the local fracture occurs. Finally the stress drops at the strain $36 \%$ and remains unchanged afterwards, which indicates the complete fracture occurs.

Some typical atomic configurations are presented in Fig. 3 for different models to capture the key pictures in the proceedings of tensile tests. Fig. 3(a) and (b) show that for the Al GB without Si impurity fracture occurs when the strain changes from $36 \%$ to $38 \%$. For the Al GB model with substitutional Si, the local fracture occurs with strain of $24 \%$ as shown in
Fig. 3(c) and (d), where the distance between $\mathrm{Al}(2)$ and $\mathrm{Al}(-2)$ in the (200) plane increases suddenly while the distance between $\mathrm{Al}(2)$ and $\mathrm{Al}(-2)$ in the (100) plane keeps unchanged. Fig. 3(e) shows that the complete fracture occurs when the strain reaches $34 \%$. For the $\mathrm{Al} \mathrm{GB}$ model with interstitial $\mathrm{Si}$, the local fracture occurs with strain of $28 \%$ as shown in Fig. 3(f) and $(\mathrm{g})$, where the distance between $\mathrm{Al}(2)$ and $\mathrm{Al}(-$ 2 ) in the (200) plane, the distance of $\mathrm{Al}(-3)$ in (200) plane and $\mathrm{Al}(-5)$ in (100) plane, and the distance between $\mathrm{Al}(-4)$ in (200) and $\mathrm{Al}(-6)$ in (100) plane increase suddenly while the distances between $\mathrm{Si}$ and $\mathrm{Al}$ atoms around it keep unchanged until the strain reaches $36 \%$ with which the complete fracture of the model occurs. It is worthy noticing that the fracture plane is not the mirror plane but the plane above it.

\subsection{Density of charge}

In the FPCTT the GBs with the substitutional and interstitial Si impurities show different fracture modes. The different modes and pictures of fracture can be further analyzed considering the change of the charge density of valence electron and density of states. The valence charge density of (100) plane of the Al GB without Si impurity, charge density of (100) and (200) planes of Al GB with substitutional $\mathrm{Si}$ and interstitial Si are plotted in Fig. 4.

Fig. 4(a) shows the density of valance charge of (100) plane of the Al GB without Si impurity with respect to the strains. When the strain is $0 \%$, the highest density value occurs between $\mathrm{Al}(2)$ and $\mathrm{Al}(-2)$, and there exists a low charge density region between $\mathrm{Al}(3)$ and $\mathrm{Al}(-3)$. When the strains are in the range of $18 \%-38 \%$, the low charge density region expands to the region near $\mathrm{Al}(2)$ and $\mathrm{Al}(-2)$ progressively with strain increasing. When the strain changes from $36 \%$ to $38 \%$ the band-shape low charge density region appears across the GB indicating that the complete fracture occurs.
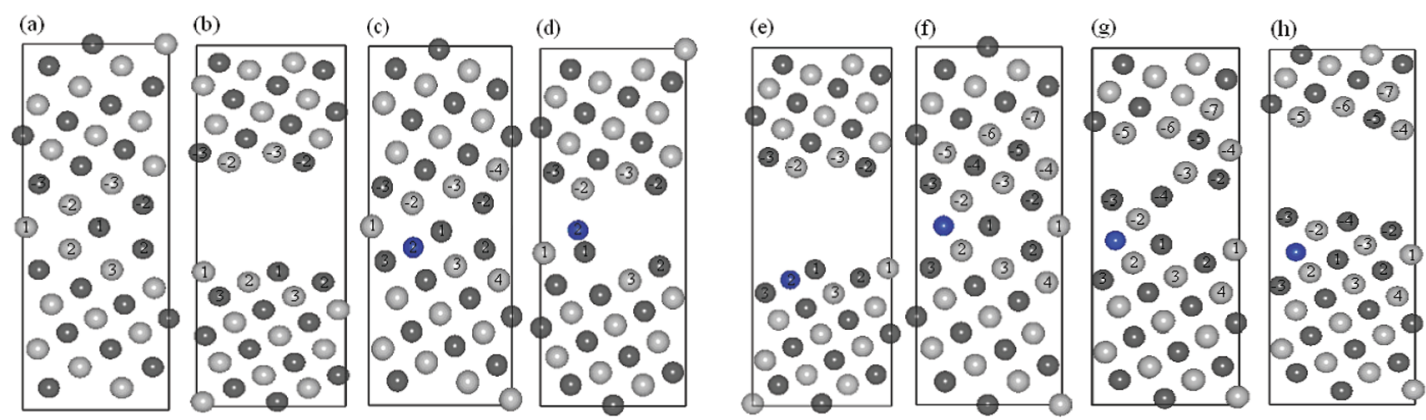

Fig. 3 Relaxed atomic configuration of (100) at the strains of $36 \%$ (a) and $38 \%$ (b) in the pure Al GB model; at the strains of $22 \%$ (c), $24 \%$ (d) and $34 \%$ (e) in the Al GB model with substitution Si; at the strains of $26 \%$ (f), $28 \%$ (g) and $36 \%$ (h) in the Al GB model with interstitial Si. Grey balls represent the Al atoms in the (100) plane, dark grey balls indicate the $\mathrm{Al}$ atoms in the (200) plane and blue balls indicate the Si atom as substitution impurity or as interstitial impurity 

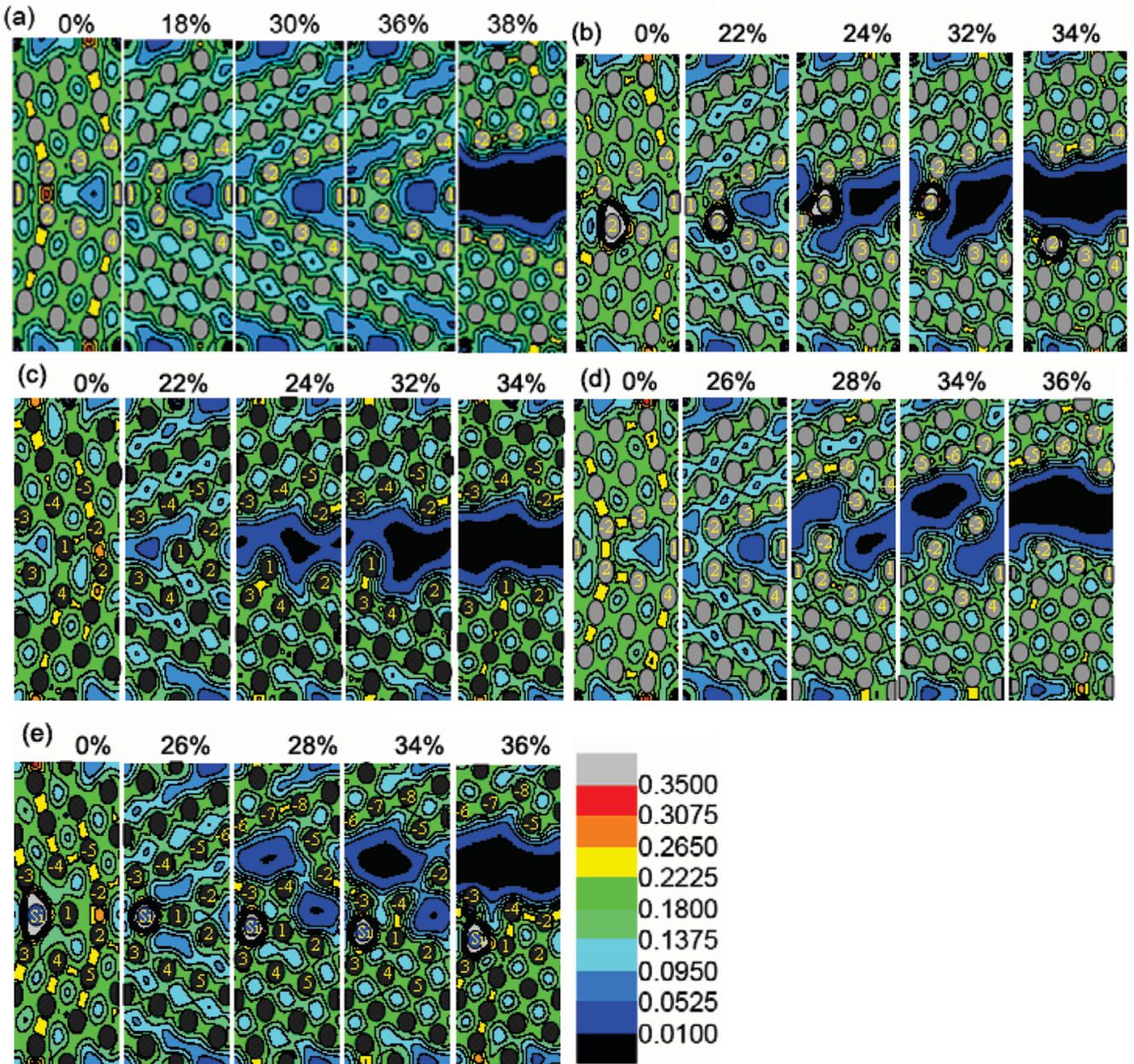

Fig. 4 Charge density distribution in the (100) plane and/or (200) plane of pure Al GB, Al GB with substitutional Si and Al GB with interstitial Si under different strains: (a) (100) plane of pure Al GB; (b) (100) plane of Al GB with substitutional Si; (c) (200) plane of Al GB with substitutional Si; (d) (100) plane of Al GB with interstitial Si; (e) (200) plane of Al GB with interstitial Si

Fig. 4(b) and (c) show the density of valance charge of (100) and (200) planes with respect to the strains in Al GB with substitutional Si. The maximum charge density value appears between $\mathrm{Si}(2)$ and $\mathrm{Al}(-2)$ in (100) plane when strain is $0 \%$, as shown in Fig. 4(b). When the strain changes from $22 \%$ to $24 \%$ the low charge density region appears in (100) plane and the band-shaped low charge density region appears across the model in (200) plane, as shown in Fig. 4(b) and (c), which correspond to the initial obvious decrease of the stress shown in Fig. 2 and the local fracture as shown in Fig. 3(d). When the strain reaches $34 \%$ the band-shaped low charge density regions appear across the model both in the (100) plane and (200) plane indicating that the complete fracture occurs.

Fig. 4(d) and (e) show the density of valence charge in the (100) and (200) planes with respect to the strains in $\mathrm{Al}$ GB with interstitial Si. When the strain changes from $26 \%$ to $28 \%$, the low charge regions appear on the up-left and up-right of the GB both in the (100) and (200) planes, which correspond to the initial decrease of the stress shown in Fig. 2 and the local fracture as shown in Fig. $3(\mathrm{~g})$. When the strain reaches $36 \%$ the band-shaped low charge density regions appear across the model both in the (100) and (200) planes indicating that the complete fracture occurs.

\subsection{Density of states}

To further analyze the effects caused by the substitutional and interstitial $\mathrm{Si}$ in the GB models of $\mathrm{Al}$ $\Sigma 5$, the local densities of states (LDOS) are calculated. In Fig. 5, the plots of the density of states for some typical atoms at $0 \%$ strain were presented, and in the following discussion we focus on the analysis of the state density of $\mathrm{s}$ band electrons.

Fig. 5(a) presents the LDOS of the atom in pure bulk Al. Fig. 5(b) shows the LDOS of $\mathrm{Al}(-2)$ in the $\mathrm{Al}$ GB without Si impurity, which is similar to the LDOS of the bulk $\mathrm{Al}$ and therefore the interaction of $\mathrm{Al}(2)-\mathrm{Al}(-2)$ is the bond with metal character.

Fig. 5(c) and (d) show the LDOS of $\mathrm{Si}(2)$ and 

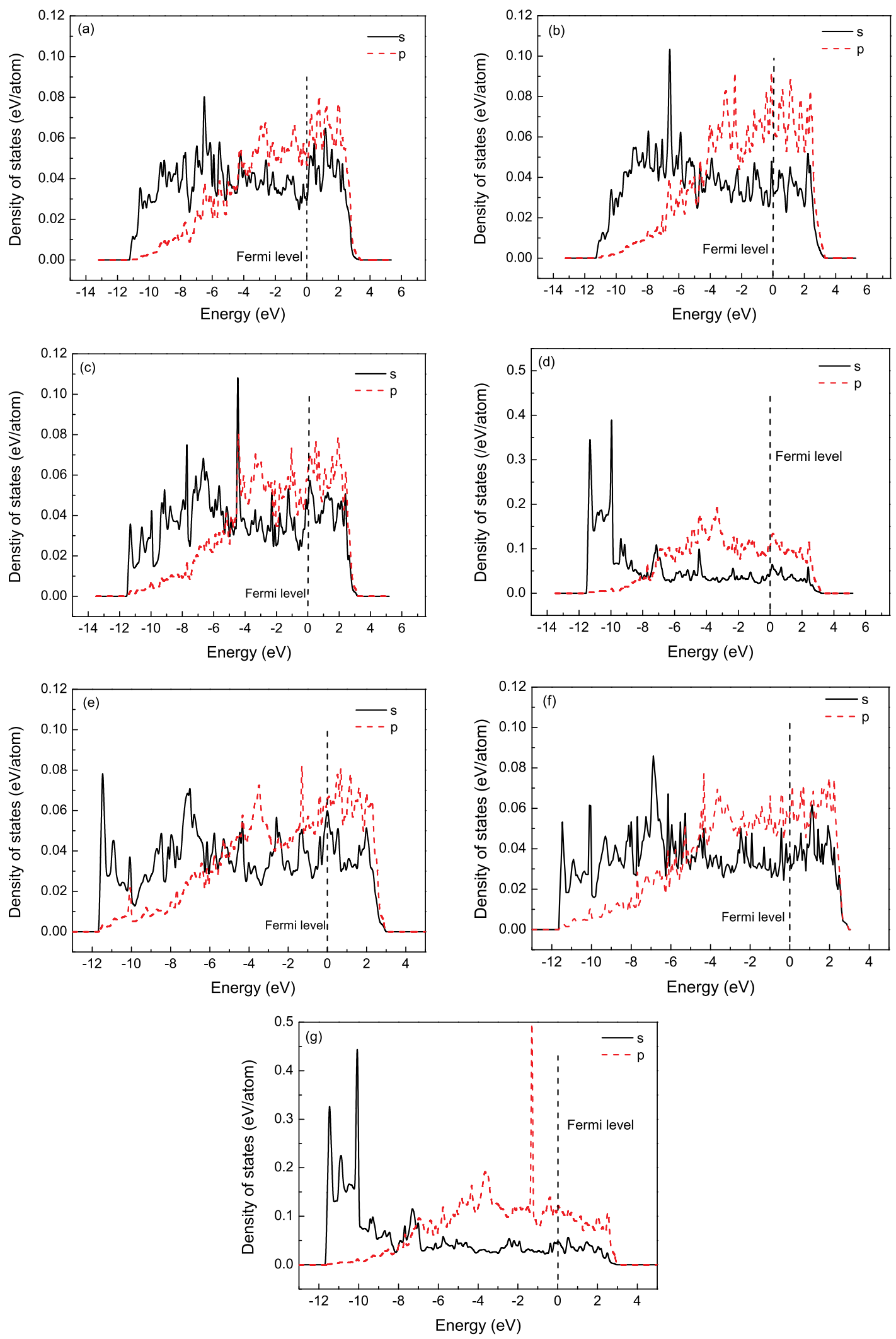

Fig. 5 Density of states for some typical atoms: (a) $\mathrm{Al}$ in pure bulk $\mathrm{Al}$; (b) $\mathrm{Al}(-2)$ on (100) plane on pure $\mathrm{Al}$ GB; (c) $\mathrm{Al}(-2)$ on (100) plane on Al GB with substitution $\mathrm{Si}$; (d) $\mathrm{Si}(2)$ on (100) plane on Al GB with substitution $\mathrm{Si}$; (e) $\mathrm{Al}(-2)$ on (100) plane on $\mathrm{Al} \mathrm{GB}$ with interstitial Si; (f) $\mathrm{Al}(-3)$ on (200) plane on $\mathrm{Al} \mathrm{GB}$ model with interstitial Si; (g) Si on Al GB with interstitial Si 
$\mathrm{Al}(-2)$ in the (100) plane of the GB with substitutional Si. Four hybridization peaks appear at -11.2 , $-10.0,-7.2$ and $-4.2 \mathrm{eV}$ for the states of $\mathrm{Al}(-2)$ and $\mathrm{Si}(2)$, indicating that the bond of $\mathrm{Si}(2)$ and $\mathrm{Al}(-2)$ is of covalent character. This bond makes $\mathrm{Si}(2)$ and $\mathrm{Al}(-2)$ in the (100) plane keep combination when the strain changes from $24 \%$ to $32 \%$ that were shown in Fig. 4(b).

Fig. 5(e), (f) and (g) show the LDOSs of $\mathrm{Al}(-2)$ in the (100) plane, $\mathrm{Al}(-3)$ in the (200) plane and $\mathrm{Si}$ in (200) plane in the GB with interstitial Si. Four hybridization peaks appear at $-11.5,-11.0,-10.0$ and $-7.2 \mathrm{eV}$ for $\mathrm{Al}(-2)$ in the (100) plane, $\mathrm{Al}(-3)$ in the (200) plane and $\mathrm{Si}$ in the (200) plane, indicating that the bonds of among them have the covalent character. The covalent-like bonds are also found between Si and other $\mathrm{Al}$ atoms around it. These bonds make $\mathrm{Si}$ and the $\mathrm{Al}$ atoms around it close combination during the whole process of tensile loading referring to that in Fig. 4(d) and (e).

According to Yuasa et al. ${ }^{[23]}$ the enhanced embrittlement of GB caused by impurity can be categorized into two kinds of mechanisms, one is the bond mobility mechanism, where the mobility of electrons is limited due to the covalent-like bonding, and the other is the decohesion mechanism, where the metal-metal bond is weakened owing to the charge transfer. From the analysis in sections 3.2 and 3.3 , it is known that these two mechanisms effect by one or one after another and the fracture modes are determined by the competition of the two mechanisms. For the Al GB model with substitutional $\mathrm{Si}$, the decohesion mechanism due to the charge transfer takes into effect firstly, which results in the appearance of low charge density region and local fracture referring to the Fig.4 (b) and although the covalent-like bonds forms between $\mathrm{Si}(2)$ and the $\mathrm{Al}$ atoms around it referring to Fig. 5(c) and (d). When the strain reaches to $34 \%$, the bond mobility mechanism takes into effect and the bond between $\mathrm{Si}(2)$ and $\mathrm{Al}(-2)$ breaks. For $\mathrm{Al} \mathrm{GB}$ model with interstitial $\mathrm{Si}$, the decohesion mechanism prevails over the bond mobility mechanism during the whole process of tensile loading and it is the dominant mechanism in this case, which results in that the fracture plane is not the mirror plane but the plane neighboring it.

\section{Conclusions}

The different fracture modes of Al $\Sigma 5$ (GB) with and without Si impurity are observed in FPCTT. The Al $\Sigma 5$ GB without Si impurity experiences merely the complete fracture in the loading process and the complete fracture plane occurs in the GB plane. For the Al $\Sigma 5$ GBs with Si impurity, the local fracture occurs before the complete fracture of the GB. The fracture mode is dominated by the competition between decohesion and bond mobility mechanisms. For the GB with interstitial Si impurity, the decohesion mechanism prevails over the bond mobility mechanism in the whole process of tensile loading. The charge density decreases in the region above the GB and weaken the interaction among the $\mathrm{Al}$ atoms in that region, which results in fracture occurring at the plane above the GB. For the GB model with the substitutional Si impurity, the decohesion mechanism takes into effect primarily and the local fracture occurs. With increasing strain the bond mobility mechanism starts to take into effect and eventually results in the complete fracture of the GB. The effect of the impurity introduced into the GB correlates to the many factors. In this study we set up the GB model only with one substitutional or interstitial Si atom at the GB.

\section{Acknowledgements}

This work was supported by National Basic Research Program of China (No. 2011CB606403) and Project of Education Department of Liaoning Province, China (No. L2010179).

\section{REFERENCES}

[1] H.Z. Ye, J. Mater. Eng. Perform. 12 (2003) 288.

[2] M. Kim, C.B. Geller and A.J. Freeman, Scr. Mater. 50 (2004) 1341.

[3] M. Yamaguchi, M. Shiga and H. Kaburaki, Science 307 (2005) 393.

[4] M. Yamaguchi, Y. Nishiyama and H. Kaburaki, Phys. Rev. B 76 (2007) 035418.

[5] G.H. Lu, Y. Zhang, S. Deng, T. Wang, M. Kohyama, R. Yamanoto, F. Liu, K. Horikawa and M. Kanno, Phys. Rev. B 73 (2006) 224115.

[6] H.H. Kart and T. Cagin, J. Achievements Mater. Manuf. Eng. 30 (2008) 177.

[7] M. Yuasa and M. Mabuchi, Phys. Rev. B 82 (2010) 094108.

[8] L. Zhang, X. Shu, S. Jin, Y. Zhang and G.H. Lu, J. Phys.: Condens. Matter 22 (2010) 375401.

[9] M. Yuasa and M. Mabuchi, J. Phys.: Condens. Matter 22 (2010) 505705.

[10] G.H. Lu, S.H. Deng and T.M. Wang, Phys. Rev. B 69 (2004) 134106.

[11] Y. Zhang, G.H. Lu, S.H. Deng and T.M. Wang, Acta Phys. Sin. 55 (2006) 2901.

[12] X.Y. Pang, N. Ahmed, R. Janisch and A. Hartmaier, J. Appl. Phys. 112 (2012) 023503.

[13] G.H. Lu, A. Suzukj, A. Ito, M. Kohyama and R. Yamamoto, Mater. Trans. 44 (2003) 337.

[14] L.H. Liu, Y. Zhang, G.H. Lu, S.H. Deng and T.M. Wang, Acta Phys. Sin. 57 (2008) 4428.

[15] S. Zhang, O.Y. Kontsevoi, A.J. Freeman and G.B. Olson, Phys. Rev. B 82 (2010) 224107.

[16] G. Kresse and J. Hafner, Phys. Rev. B 49 (1994) 14251.

[17] G. Kresse and J. Furthmüller, Phys. Rev. B 54 (1996) 11169.

[18] G. Kresse and J. Furthmüller, Comput. Mater. Sci. 6 (1996) 15.

[19] P.E. Blöchl, Phys. Rev. B 50 (1994) 17953.

[20] G. Kresse and D. Joubert, Phys. Rev. B 59 (1999) 1758.

[21] J.P. Perdew, K. Burke and M. Ernzerhof, Phys. Rev. Lett. 77 (1996) 3865.

[22] J.P. Perdew, K. Burke and M. Ernzerhof, Phys. Rev. Lett. 78 (1997) 1396.

[23] M. Yuasa and M. Mabuchi, Adv. Mater. Res. 409 (2012) 455. 